\title{
A Novel Configuration for Superconducting Space Radiation Shields
}

\author{
V.Calvelli, R.Musenich, F.Tunesi and R.Battiston
}

\begin{abstract}
Long time exposure to Galactic Cosmic Rays is one of the most problematic threats for a manned mission in the deep space. In the last decades, several studies of active, superconducting magnetic shields were performed leading to the proposals of apparently promising magnetic configurations. However, as the interaction of energetic particles with the materials composing the magnets was not taken into account, the contribution of secondary particles to the astronauts' radiation dose was neglected and consequently the actual effectiveness of the shields was overestimated. In the frame of the EU-FP7-SR2S project, a study of superconducting space radiation shield was performed associating the optimization of the magnetic configuration with Monte Carlo simulation of the dose reduction. It was found that most of the magnetic shields proposed in previous works are not adequate and a novel configuration, transparent to radiation, was proposed based on toroids arranged with their axes perpendicular to the spacecraft axis. Compared to other shields with the same shielding power, such a configuration results in lower magnetic field at the superconductor and very light design.
\end{abstract}

Index Terms-Superconducting magnets, space technology, cosmic rays.

\section{INTRODUCTION}

A well-known problem to face in the future, long-term, manned missions in deep space is ionizing radiation. Solar particle events (SPE) and Galactic Cosmic Rays (GCR) will threat the health of astronauts exposing them to several kinds of late and acute diseases, even fatal [1]. Several solutions have been proposed to protect crews, based on either energy loss in the interaction with matter (passive shielding) or the deflection of particles by means of magnetic fields (active shielding). Passive shielding requires thick layers of materials around the spacecraft cabin resulting in large mass increasing. Active shielding is a valid alternative but requires huge superconducting magnets that have to operate in space for years. It would have been unthinkable 55 years ago when

Manuscript received 02 September 2016

Valerio Calvelli is with Istituto Nazionale di Fisica Nucleare (INFN), Sezione di Genova, and Dipartimento di Fisica, Università di Genova, via Dodecaneso 33, 16146 - Genoa, Italy (phone: +39 010353 6445; e-mail: valerio.calvelli@ge.infn.it).

Riccardo Musenich, is with Istituto Nazionale di Fisica Nucleare (INFN), Sezione di Genova.

Filippo Tunesi is with CGS SpA, Milan, and with Politecnico di Milano, Milan, Italy.

Roberto Battiston is with INFN-TIFPA, with Dipartimento di Fisica, Università di Trento, Trento, Italy and with Italian Space Agency (ASI), Rome, Italy. the magnetic shielding principle was first proposed [2], but now, thanks to the progress in magnet technology and to the development of high temperature superconductors as well as of magnesium diboride, active shielding has become a possible option.

The existing literature reports several studies about magnetic shielding of space radiation, however until recent years all of them have based the evaluation of shielding efficiency only on the deflection by means of magnetic field, neglecting the interaction of particle with the materials composing the magnet. Materials act as passive shielding, stopping part of the incoming charged particles, however the interactions also generate secondary particle showers, which give an additional contribution to the astronauts' radiation dose. A significant part of the secondary particles is neutrons, which cannot be magnetically shielded. Recent studies have shown that the particle-material interactions results in a reduction of the effective shielding power [3].

Magnetic systems for particle shielding are large superconducting magnets positioned around the spacecraft habitat; the most studied configurations are solenoids and toroids. Solenoids can be arranged in two ways: one large solenoid hosting the cabin within its bore or several solenoids positioned circularly around the spacecraft. In the toroidal configuration, the spacecraft cabin is hosted within the bore of the toroid. While the fringe field is null inside the bore of an ideal toroid, solenoidal configurations require an inner solenoid to cancel the stray field in the habitable module. The three configurations are shown in Fig. 1. In all of them, a large amount of material is distributed around the spacecraft generating secondary particle showers when hit by energetic cosmic rays.

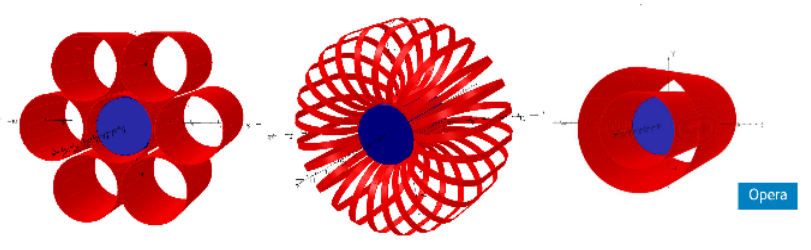

Fig.1. Three "classical" configurations for space radiation shielding. On the left, six solenoids surround the cabin (blue area); in the middle a coaxial toroidal configuration, on the right two concentric solenoids surround the habitat and compensate their magnetic fields inside the habitat itself.

The necessity to make the shield lighter and to reduce the secondary particle production triggered the search for a new magnetic configuration. 


\section{MAGNETIC SYSTEM}

A toroidal magnet can be used as magnetic shield not only in the configuration shown in Fig.1 but also as if particles

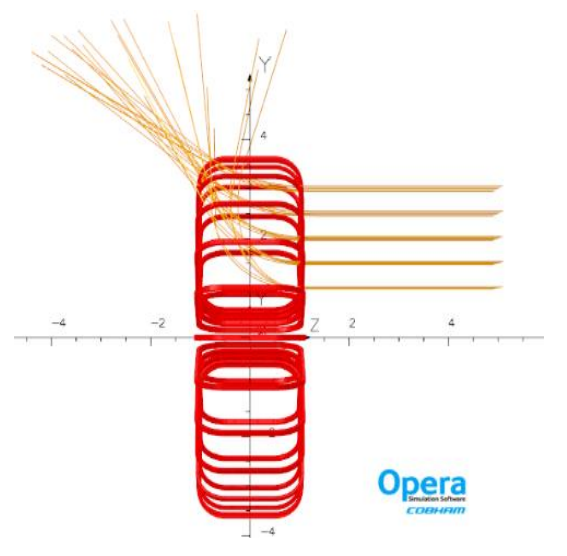

Fig.2. A magnetic lens deflecting incoming charged particle

move parallel to the axis. In 2000, a shield to protect astronauts against SPE, based on a toroidal magnetic lens, was proposed by Spillantini et al. [4]. Later, toroidal magnetic lens were used as end caps of toroidal magnetic shields [5, 6].

The novel configuration is based on a set of toroidal magnets positioned around the habitable module in such a way their axes are directed radially. It must be noted that the field of an ideal toroid is fully confined; therefore, a set of quasiideal toroids leaves many unshielded areas between them. In order to guarantee shielding all around the cabin the field must to be not confined, consequently the number of coils of each toroid has to be chosen low enough. After an optimization of the magnetic configuration, it was found that 3 coil toroids is the best solution. The number of toroids composing the shield depends on the maximum allowable dimension and on the spacecraft size. Fig. 3 shows a particular arrangement with 4 toroids, positioned at about $3.5 \mathrm{~m}$ from the spacecraft axis.

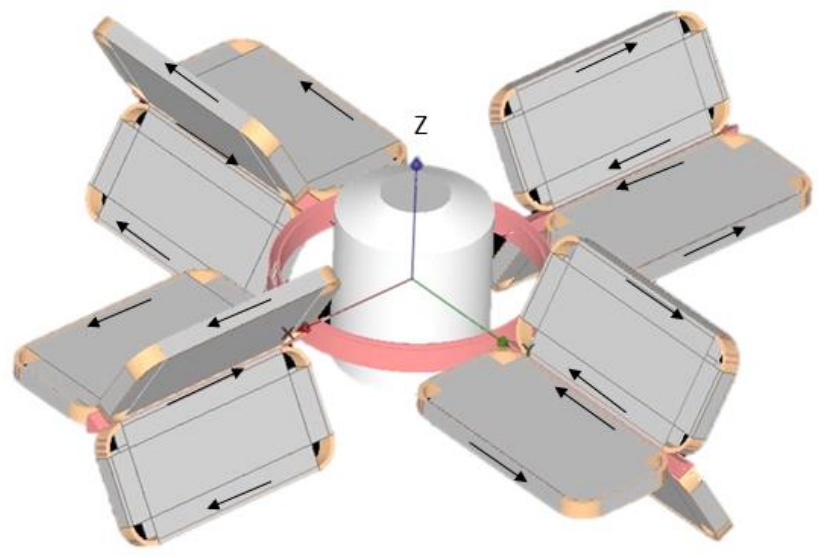

Fig.3. Magnet system surrounding the spacecraft habitat (gray cylinder). Part of the mechanical structure is not shown. Black arrows indicate the direction that the current should have to produce the shielding effect.

Each toroid is composed by three coils, $120^{\circ}$ apart (one of the coil lies on the xy plane, the others are symmetrically tilted by $60^{\circ}$ ). Despite its spread, the magnetic field within the cabin is kept low enough to affect neither the astronauts' safety, nor instrument operation. Such a new shielding configuration has major advantages respect to the co-axial toroid: it does not need a massive inner structure as discussed in next section and its mass is distributed in such a way to less interact with incoming charged particles. Consequently, it generates less secondary particles.

The dimensions of the radiation shield have to be defined on the basis of both the spacecraft characteristics and the launcher size. A possible configuration was evaluated by hypothesizing the use of the Columbus module of the

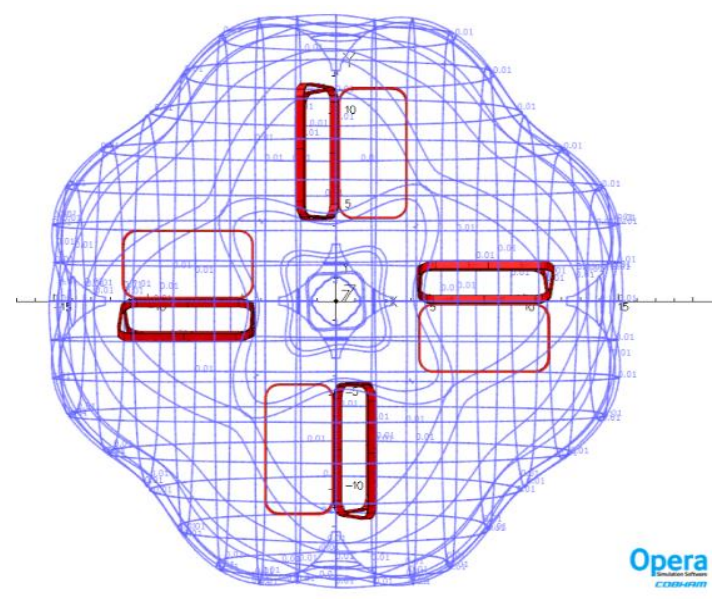

Fig.4. Magnetic flux density $(10 \mathrm{mT})$ contour lines of the new configuration. The fringe magnetic field has a major role to cover the volumes outside the toroids, furnishing almost a $4 \pi$ shield.

International Space Station as habitable cabin, which is $7 \mathrm{~m}$ long and $4.5 \mathrm{~m}$ in diameter. Due to its very large dimension, the magnetic system is designed to be modular, namely, to be assembled in orbit after having separately launched its parts (magnets and inner support). Consequently, the size of the toroidal magnets was set to fit the fairing of the future heavy launcher SLS Block 1B Cargo [7].

The characteristics of the shield are summarized in table I.

TABLE I.

Characteristic OF THE SuPERCONDUCTING SHIELD

\begin{tabular}{ll}
\hline Coil length & $7.5 \mathrm{~m}$ \\
Coil width & $3 \mathrm{~m}$ \\
Total current per toroid & $8.9 \mathrm{MA}$ \\
Maximum field at the conductor & $6.5 \mathrm{~T}$ \\
Magnetic flux density in the cabin & $<4.5 \mathrm{mT}$ \\
Mass of the winding & 31 tons \\
Total mass & 54 tons \\
Stored energy & $800 \mathrm{MJ}$ \\
\hline
\end{tabular}

In Fig. 4, the contour lines clearly show that the magnetic field is confined within the whole magnetic system and is spread all around the cabin. Due to the shape of the field, the new non-axial configuration was nicknamed "pumpkin configuration".

Monte Carlo simulations of the equivalent radiation dose due to GCR show that the shielding power of the described configuration is equivalent to that of a toroid in co-axial configuration having a total mass of at least 80 tons. The equivalent dose due to GCR is about $55 \%$ respect to that 
adsorbed by an astronaut within a cabin without any protection [8].

Differently, charged particles generated by the Sun are not enough energetic to pass through the magnetic shield that consequently offers a complete protection of the habitat also during intense solar events like coronal mass ejections and solar flares.

\section{MechanicAl StRUCTURE}

The coils of toroids are subject to inward forces, i.e. radially directed to the axis. In the case of a toroid surrounding the spacecraft habitat hosted in its bore, a large mechanical structure is required, strong enough to prevent any risk for the cabin. In the pumpkin configuration, the inner radius of the toroids is small; therefore, the large inward forces experienced by the three coils of each toroid are easily supported by a central bar. Differently, the total radial forces experienced by the toroids, i.e. the forces along $\mathrm{x}$ and $\mathrm{y}$ axes shown in Fig.2, are relatively weak and directed outward. To support the outward forces, the inner beam of each toroid is connected to a mechanical structure surrounding the cabin. The forces acting on the winding are kept by grids of ropes.

Specific attention must be payed analyzing the azimuthal forces, which result in bending momenta acting on the axis of each toroid. Consequently, a mechanical support is necessary to limit the axis deformation. Such a support is also used to hold forces and torques due to slight construction asymmetries.

TABLE II.

Magnetic Forces Acting on Superconducting Shield

\begin{tabular}{ll}
\hline Radial outward force per toroid & $370 \mathrm{kN}$ \\
Azimuthal net force per toroid & $27 \mathrm{kN}$ \\
Force toward toroid axis (per coil) & $43.5 \mathrm{MN}$ \\
\hline
\end{tabular}

An evaluation of the torque due to possible asymmetry between the coils was done by supposing both non-uniform current distribution and magnet/coil misplacing. A $10 \%$ reduction of the full operation current in one of the magnets will generate forces between it and the two adjacent magnets which results in torques around the $\mathrm{z}$ axis of $184 \mathrm{kNm},-160 \mathrm{kNm}$ and $-24 \mathrm{kNm}$. The mechanical structure can support small current differences but cannot bear the full discharge of a single coil when the others are operating at full current. Consequently, two solutions are possible: the first is to series connect the magnets; the second is to simultaneously charge/discharge the magnets. Of course, if the magnets are not series connected, in case of quench of one of them, the quench protection system has to fast discharge the others.

Despite each toroid will constructed and assembled on Earth, the entire structure is designed to be assembled in orbit; therefore, small errors in magnet positioning must be taken into account. The torque experienced by a magnet azimuthally misplaced by $1 \mathrm{mrad}$, i.e. respect to $\mathrm{z}$-axis, is about $12 \mathrm{kNm}$. The torques due to angular misplacing around the other axes are lower, being limited to a maximum value of $5 \mathrm{kN} / \mathrm{mrad}$.

The result of the mechanical analysis is that the pumpkin configuration requires a lighter mechanical structure respect to a co-axial toroidal shield. A schematic view of the mechanical structure is shown in fig 5 .

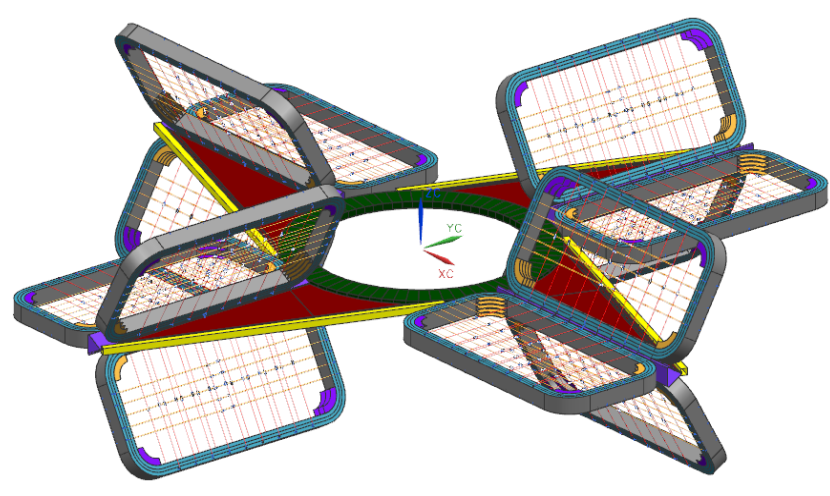

Fig.5. Schematic view of the shield mechanical structure.

\section{CONDUCTOR}

The two main requirements of a space-born magnet are reliability and lightness. The former is necessary because a failure could result in aborting of the mission while the latter has impact on both costs and trip duration. Consequently, the conductor must be chosen bearing in mind the abovementioned requirements. The conductor designed for the SR2S magnet is a $0.6 \mathrm{~mm}$ thick, $4.8 \mathrm{~mm}$ wide Titanium clad $\mathrm{MgB}_{2}$ tape with a $1.1 \mathrm{~mm}$ thick Aluminium strip bonded on it. The mass density of the $\mathrm{Ti}-\mathrm{MgB}_{2}$ tape is $4000 \mathrm{~kg} / \mathrm{m}^{3}$ making it the lightest among the superconducting conductors. The Aluminium strip must be added to protect the magnet in case of quench. The reliability of the magnet is related to both stability and quench protection. The use of an $\mathrm{MgB}_{2}$ based conductor allows operating the magnet with higher enthalpy margin respect to low temperature superconductors; therefore, it guarantees the magnet stability. On the contrary, the quench propagation velocity is reduced by high values of the specific heat capacity. Consequently, the higher the temperature is, the more difficult the magnet protection is and an active quench protection system is mandatory. In order to limit the magnetic energy density and to allow protection in case of quench, the overall current density is set at $80 \mathrm{~A} / \mathrm{mm}^{2}$.

The details of the conductor design and prototype production are described in [9].

\section{CONCLUSiOnS}

Long duration manned missions in deep space require adequate strategies to protect astronauts against cosmic rays hurts. Active shielding based on superconducting magnets placed around the habitable cabin is an interesting and promising way to solve the issue (Fig.6). In last years, the magnetic configurations proposed in the past were deeply studied pointing out a major problem: the generation of secondary particles due to the impact of cosmic rays on the magnet materials partially neutralizes the shielding effect of the magnetic field.

To minimize the production of secondary particles a new shield configuration based on toroids disposed in a non-axial arrangement was studied. Monte Carlo simulation showed that a reduction of a factor of two of the dose due to GCR is possible with a relatively light structure, compared to axially 
arranged toroids. Moreover, while a toroid around the spacecraft require a robust inner mechanical structure to support the inward forces, in the new configuration the magnetic forces acting on each toroid are directed outward.

Such a shield can be coupled to layers of low atomic number materials in order to further reduce the dose by stopping high energy ions.

Despite the reduction of the dose due to GCR is not dramatic, it is enough to reduce the dose adsorbed during a trip to Mars to acceptable values. Furthermore, it must be highlighted that the shield is able to fully deflect solar particles, protecting astronauts also during intense solar events.

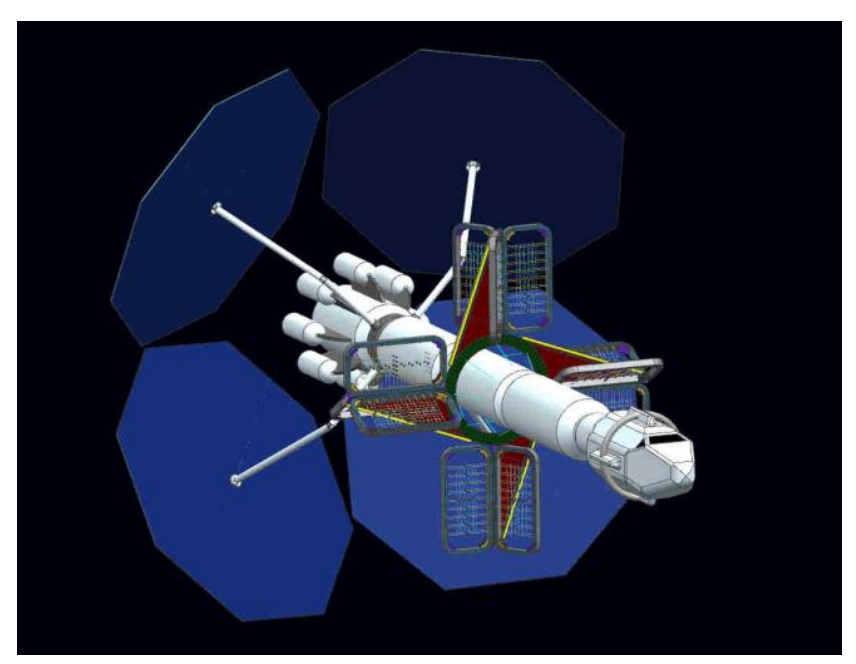

Fig.6. Artistic view of a spacecraft equipped with the magnetic shield. Debri protection and cryogenic shields are not shown. (Courtesy of Compagni: Generale dello Spazio)

\section{ACKNOWLEDGMENT}

The described study was co-funded by the EU FP7 SR2S Project and by INFN. The authors wish to thank all the partners of the SR2S project: INFN, CEA-Saclay, CERN, Compagnia Generale dello Spazio (CGS), Thales-Alenia Space Italia, Columbus Superconductors and Carr Communication.

\section{REFERENCES}

[1] F.A.Cucinotta and M.Durante, "Cancer Risk from Exposure to Galactic Cosmic Rays: Implications for Space Exploration by Human Beings" Lancet Oncol. 7, 431-435, 2006.

[2] R.H. Levy, Radiation Shielding of Space Vehicles by Means of Superconducting Coils, ARS Journal, 31, 11, 1568-1570, 1961.

[3] M.Vuolo, M.Giraudo, R.Musenich, V.Calvelli, F.Ambroglini, W.J.Burger, R.Battiston, "Monte Carlo simulations for the space radiation superconducting shield project (SR2S)", Life Sciences in Space Research, 8, 22-29, 2016

[4] P.Spillantini, F.Taccetti, P.Papini, L.Rossi, "Radiation shielding of spacecraft in manned interplanetary flights", Nuclear Instruments and Methods in Physics Research, A 443, 254-263, 2000.

[5] Hoffman, J.A., Fisher, P. and Batischev, O., "Use of Superconducting Magnet Technology for Astronaut Radiation Protection", NIAC Phase I Final Report, Contract CP 04-01, 2005.

[6] R.Musenich, V.Calvelli, S.Farinon, W.J.Burger, R.Battiston, "Space Radiation Superconducting Shields" Journal of Physics: Conference Series, 507, 032033, 2014.

[7] www.nasa.gov/exploration/systems/sls/sls-vehicle-evolution.html.

[8] M.Vuolo, M.Giraudo, R.Musenich, V.Calvelli, F.Ambroglini, W.J.Burger, R.Battiston, "Monte Carlo simulations results in novel superconducting radiation shield structure" - to be published.

[9] R.Musenich, D.Nardelli, S.Brisigotti, D.Pietranera, M.Tropeano, A.Tumino, V.Cubeda, V.Calvelli, G.Grasso, "Ti-MgB2 Conductor for Superconducting Space Magnets" IEEE Trans. On Appl. Supercond., 26 (4), 6200204, 2016 